2

3 \section{(1)}

Nicolas Robin* ${ }^{1}$, Guillaume R. Coudevylle $*^{2}$, Olivier Hue ${ }^{3}$, and Lucette Toussaint**.

1 Email: robin.nicolas@hotmail.fr

2 Email: guillaume.coudevylle@univ-antilles.fr

3 Email: ohue@univ-ag.fr

*Université des Antilles; Laboratoire "Adaptation au Climat Tropical, Exercice \& Santé"; Faculté des Sciences du Sport de Pointe-à-Pitre, France. Tel +(0033)5 90483173 ; Fax $+(0033) 590483179$

**Université de Poitiers; Université François Rabelais de Tours; Centre National de la Recherche Scientifique; Centre de Recherches sur la Cognition et l'Apprentissage. Tel +(0033)5 49454698 ; Email: lucette.toussaint@univ-poitiers.fr 4

Correspondence concerning this article should be addressed to Nicolas Robin, Laboratoire

"Adaptation au Climat Tropical, Exercice \& Santé" (UPRES EA 3596), Campus Fouillole, BP 592, 97159, Pointe à Pitre Cedex, France.

Contact: robin.nicolas@hotmail.fr 


\title{
The Influence of Tropical Climate on Imagined Walking Time
}

\begin{abstract}
This study investigated the effects of a Tropical Climate (TC) on actual and imagined walking times. Participants had to execute and imagine walking 3 distances with or without a 10-kg weight in either a TC or an Air Conditioning (AC) condition. The motor imagery quality was evaluated by computing the isochrony index. The results revealed that movement times were shorter for imagined walking than for actual walking and increased with the distance of the paths in both cases. By contrast, the effect of the load increased actual walking times for the 10- and 15-meter distances without affecting imagined walking times.

Importantly, the isochrony index was negatively affected by the increase in load and distance and the effects of distance were amplified in a TC. These findings showed that the environmental climate in which motor imagery arises can modulate the subject's ability to build up the temporal characteristics of simulated actions.

Keywords: mental chronometry, motor imagery, tropical environment, walking time
\end{abstract}




\section{The Influence of Tropical Climate on Imagined Walking Time}

Motor Imagery (MI) is the mental representation or cognitive rehearsal of a motor act or movement in the absence of any overt motor output (Williams, Guillot, Di Rienzo, \& Cumming, 2015). Given its importance in MI training programs, the timing of imagined movements has received a growing body of attention (Guillot, Hoyek, Louis, \& Collet, 2012). However, most studies were realized in neutral climate while other environments, like tropical climate that concerned millions of people, are less concerned. The main objective of this study was to evaluate the influence of tropical climate on imagined walking time.

MI is a frequently employed cognitive strategy that is used to facilitate motor learning (Goss, Hall, Buckolz, \& Fishburn, 1986), relearning (Malouin, Richards, Jackson, \& Doyon, 2010), muscle strength (Lebon, Collet, \& Guillot, 2010), sport performance (Robin

Dominique, Toussaint, Blandin, Guillot, \& Le Her, 2007), rehabilitation (Rulleau, Mauvieux, \& Toussaint, 2015) and confidence or motivation (see Guillot \& Collet, 2008; Short, Tenute, \& Feltz, 2005 for reviews). A number of experiments have argued for a functional and anatomical correspondence between MI and actual movement (Jeannerod \& Frak, 1999). Indeed, neuroimagery studies found that some neural structures (i.e., parietal cortex, supplementary motor area, premotor cortex, prefrontal cortex, cerebellum, primary motor cortex and spinal cord) are similarly involved in MI and actual movement execution (Batula, Mark, Kim, \& Ayaz, 2017; Decety, 1996; Gerardin et al., 2000; Jeannerod, 2001). According to Cerritelli et al. (2000), the main difference between the functional neuroanatomy of imagined and real actions is that the magnitude of activation is reduced during MI. Psychophysical experiments have shown that MI preserves the same spatio-temporal characteristics and obeys the same laws of movement control as do actual movements (Cerritelli, Maruff, Wilson, \& Currie, 2000; Papaxanthis, Pozzo, Skoura, \& Schieppati, 2002). The similarity between actual and imagined performance would be at the origin of the 
improvement of motor performance in motor learning studies (Jeannerod, 2001). However, some studies have shown that MI can be specifically affected by factors such as sex, age, imagery ability, imagery modality and imagery conditions (see Holmes \& Calmels, 2008 for a review). In the present experiment, we were interested in the effect of climatic conditions on the temporal features of imagined walking in healthy young adults. We specifically examined whether the impact of climatic conditions (tropical climate vs. air conditioning) varied as a function of task constraints (distance of walking and load).

Several authors have previously examined the effects of distance/amplitude of movements on motor imagery. Some have reported a temporal equivalence between actual and imagined actions for graphic movements (Decety \& Michel, 1989; Papaxanthis et al., 2002), pointing (Maruff \& Velakoulis, 2000) and walking (Decety, Jeannerod, \& Prablanc, 1989; Papaxanthis et al., 2002) using the mental chronometry paradigm. The concept of mental chronometry refers to the time needed to process sensory-motor tasks to infer the content and the temporal sequencing of cognitive operations (Guillot et al., 2012). The latter added that the mere comparison of the time taken to actually perform versus mentally imagine the same movement provides substantial information about the individual ability to preserve the temporal organization of the actual performance during MI. Some studies used the isochrony index (absolute difference between the average duration of the executed and imagined walk) in order to observe if MI was closed to real actions, but most studies compared MI and actual movements times in different conditions (Guillot \& Collet, 2005). For example, Decety et al. (1989) have shown that both actual and imagined walking duration increased with distance (5,10 and 15 meters) and took almost the same time whatever the distance. By contrast, other authors have reported that participants might have difficulty in preserving the temporal characteristics of imagined movements. It has been shown that the accuracy of MI time decreased for long task durations (Grealy \& Shearer, 2008; Guillot \& 
1 Collet, 2005; Hanyu \& Itsukushima, 2000), whereas it increased for short movements

2 (Calmels, Holmes, Lopez, \& Naman, 2006; Orliaguet \& Coello, 1998). Increased MI time was also observed when participants carried a $25-\mathrm{kg}$ weight on their shoulders (Decety et al., 1989). The effect of load on MI time was confirmed by Cerritelli et al. (2000) with a 2-kg weight attached to the top of a pencil during a pointing task. The authors argued that the increase in imagined movement duration illustrated the disturbance of the force calculation component induced by weight. Decety et al. (1989) hypothesised that this increase in MI time occurred because participants programmed a greater muscle force in order to overcome the additional weight when moving. According to Ceritelli et al. (2000), when participants physically walked, this additional force allowed them to maintain the same speed carrying the weight as when no weight was carried. However, Corcos (1994) proposed that as increases in force are generally associated with increased movement duration, the additional force programmed when participants realized MI with the weight was interpreted as representing that the duration of their movements should be increased. In addition, Papaxanthis, Schieppati, Gentili, and Pozzo (2002) proposed that, during imagined loaded walks, the increase in the programmed force was not used to overcome any resistance, as was the case for the actual performed loaded walks, and consequently participants transferred the 'required' increase in force into an increase in movement duration.

What about the duration of MI for situations frequently encountered in everyday life? It is generally advised not to carry more than $10 \mathrm{~kg}$ in a school bag or a rucksack for hiking, for example. Papaxanthis et al. (2002) observed that participants could accurately mentally simulate an accustomed weight. Indeed, using an arm movement task with or without an added load of 1 and $1.5 \mathrm{~kg}$, these authors showed that the actual movements significantly increased in duration as a function of mass. Moreover, they observed that the duration of the imagined movements was very similar to that of the actual movements regardless of the 
subject and the load condition. These results show that both inertial and gravitational constraints are accurately incorporated into the timing of the MI process.

Because all these experiments were conducted in standard conditions (i.e., under a temperate climate), we specifically investigated the effect of a Tropical Climate (TC vs. AC for Air Conditioning) on MI, using a walking task performed at different distances $(5,10$ and 15 meters) with or without a $10-\mathrm{kg}$ weight in a rucksack. The TC is a hot and wet climatic environment (Hue, 2011), characterized by a high level of relative humidity exceeding $70 \%$ relative humidity $(\mathrm{rH})$, in which temperatures exceeding $31^{\circ} \mathrm{C}$ can be considered environmental stressors. Heat stress is generally associated with decreased performance for multiple cognitive and motor tasks (Berg et al., 2015; Gaoua, 2010; Qian et al., 2015; Robin, Coudevylle, Hue, \& Sinnapah, in press; Wetsel, 2011), and the physiological responses of the human body to heat have been documented and modeled (Hancock \& Vasmatzidis, 2003). However, the effects of heat stress and more precisely of a TC on cognition are less well understood. Some works have reported that simple mental tasks showed little, if any, decrement in the heat. In contrast, more difficult tasks showed the onset of decrements in the range between 30 and $33^{\circ} \mathrm{C}$, regardless of the duration of exposure (Ramsey \& Kwon, 1992). Gaoua (2010) found that working memory was impaired during heat exposure without alteration in the attentional processes and proposed that impairments in cognitive function with passive hyperthermia are task dependent. The author suggested that exposure to a hot environment is a competing variable for the cognitive processes. Heat stress seems to be able to decrease cognitive performance for different tasks but remains poorly understood in the mental chronometry domain. It has, however, been shown recently that a Tropical Climate (TC) interacts with imagery ability and modulates MI performance in comparison to Air Conditioning (AC) (Robin et al., in press). Indeed, using the Vandenberg and Kuse Mental Rotation Test (VMRT) and the Movement Imagery Questionnaire Revised version (MIQ-R; 
1 Hall \& Martin, 1997), the authors revealed that poor imagers had worse VMRT scores in a

2 TC than in AC, whereas no significant difference was observed for good imagers. Moreover,

3 working memory and frontal cortex, that are also solicited during MI (Pearsons, Naselaris,

4 Holmes, \& Kosslyn, 2015), are negatively affected by heat (Gaoua, Racinais, Grantham, \& El

5 Massioui, 2011). However, to date, very few are known about the effect of a TC on a

6 subject's ability to build up the temporal characteristics of a simulated action.

With this shortcoming in mind, the aim of the present experiment was to evaluate whether a TC influenced the mental representation of walking time. As previously suggested, the effect of a TC on cognitive processes may be task dependent. If this is the case, then it may be that the imagined walking times would be negatively affected by the deleterious effect of a TC, particularly when task constraints increase, as when the distances of paths become longer and when a load is added on the participants' shoulders.

\section{Methodology}

\section{Participants}

One hundred seventy-nine participants (40 females and 139 males), self-declared to be right-handed, gave their informed consent to participate in the study $\left(M_{\mathrm{age}}=18.78\right.$ years, $S D=$ 0.94). All were Guadeloupian students at the University of Antilles who lived in the tropical environment throughout the year. They had normal or corrected-to-normal vision and no history of motor or neurological disorders and were naïve to the aim of the study. As illustrated in Table 1, the participants were randomly split (computer-generated list) into four independent groups involving two load conditions (loaded vs. unloaded) and two climate conditions (AC vs. TC). No subject was aware of the specific hypotheses of the study or had previous experience or explicit knowledge concerning motor imagery processes. The experiment was both noninvasive and performed in accordance with the ethical standards established by the Declaration of Helsinki. 


\section{Please insert Table 1 about here}

\section{Materials and Tasks}

Participants were asked to walk or to imagine walking at a self-selected speed along straight paths of 5, 10 and 15 meters ( 6 trials by distance of paths in a randomized order). The walking times were measured in both the executed and the imagined conditions. The executed performance of each walk was recorded by the researcher, who started an electronic stopwatch (Geonaute ONstart 100, temporal resolution $1 \mathrm{~ms}$ ) at the onset of the movement and stopped it once the movement was finished. It is important to note that the temporal measurement with a manual stopwatch can never be that accurate (1 ms), due to human inaccuracy. Participants recorded the duration of their imagined performance by starting and stopping the stopwatch for themselves when they began and finished the imagined walk. They were instructed to imagine themselves, from an internal first-person perspective, walking to the path. The difference between internal imagery (experiencing themselves moving) and external imagery (watching themselves perform the action) was clearly explained and all participants understood that they were to perform internal motor imagery. Finally, during imagery, participants remained standing and closed their eyes.

\section{Procedure}

Before the experiment, all the participants completed the MIQ-R (Hall \& Martin, 1997), which is an 8-item self-report questionnaire assessing individual movement imagery abilities. Participants performed, then imagined and finally rated their imagery experience of four different movements. During MI, participants were asked to either "see" (visual imagery) or "feel" (kinesthetic imagery) one of the four movements. Following this, they were instructed to rate the ease or difficulty of imaging the movement on a 7-point Likert scale (1hard to imagine, 7-very easy to imagine). Thus, participants imagine each of the four movements, by focusing either on the visual modality or the kinesthetic modality separately 
1 by instruction, resulting in a final rating of items. With reference to the Likert scale, the

2 maximal score (for higher MI abilities) could be 28 and the minimum could be 4 for each 3 imagery modality.

Then, the participants performed the main experiment in a rectangular room $(20 \times 7$ meters). Participants performed the actual walking task first and the imagined walking task second, for the 3 paths (randomly distributed). Then actual and imagined walking tasks and target distances were randomly distributed in order to avoid block effects. They performed both the actual and the imagined walking task either in the $\mathrm{AC}$ condition (i.e., mean temperature $=24.13^{\circ} \mathrm{C}, S D=0.75$; humidity $\left.=45.4 \% \mathrm{rH}, S D=3.2\right)$ or in the $\mathrm{TC}$ condition (i.e., mean temperature $=31.26^{\circ} \mathrm{C}, S D=0.45$; humidity $=73.5 \% \mathrm{rH}, S D=7.9$ ). Temperature and humidity were recorded with a Fisherbrand thermohygrometer (humidity precision $\pm 5 \%$ $\mathrm{rH}$ from 35 to $75 \% \mathrm{rH}$ and $\pm 8 \% \mathrm{rH}$ beyond; temperature precision $\left.\pm 0.1^{\circ} \mathrm{C}\right)$. In each climatic condition, participants performed the executed and the imagined task with (loaded condition) or without (unloaded condition) a 10-kg load placed in a rucksack on their shoulders.

Whatever the load condition, the participants completed 36 trials (18 executed and 18 imagery trials, 6 trials for each of the 3 paths) and had 1-minute rest after 12 trials.

\section{Data analysis}

A first analysis of the MIQ-R scores was used to verify the homogeneity of the imagery ability between the groups according to the climatic and load conditions. Separate non-parametric Kruskal-Wallis tests were performed on the visual and kinesthetic imagery scores for the groups (AC-unloaded vs. AC-loaded vs. TC-unloaded vs. TC-loaded). Because of the use of independent groups, more good imagers in a group and more poor imagers in another group would make it difficult to interpret the results.

A second analysis was used to examine the effects of load, climate and distance of paths on the duration of the actual and imagined walks. An ANOVA was performed on the 
movement times with climatic (TC vs. AC) and load (loaded vs. unloaded) conditions as between-participants factors and distance of paths (5,10 and $15 \mathrm{~m})$ and action (actual or imagined) as within-participants factors.

We then examined motor imagery quality by computing the isochrony index, which corresponds to the absolute difference between the average duration of the executed and imagined walk for each trial. The isochrony index makes it possible to observe, for a participant, if what he does in MI is close to what he does in physical practice. A value close to zero indicates good imagery ability. A low value indicates that participants have the facility to build up the temporal characteristics of the simulated action, whereas a high value indicates greater difficulty. An ANOVA was performed on the isochrony index with the climatic (TC vs. AC) and load (loaded vs. unloaded) conditions as between-participants factors and the distance of the path $(5,10$ and $15 \mathrm{~m})$ as a within-participants factor. We ensured that the movement times and the isochrony index were normally distributed using the KolmogorovSmirnov test before performing the ANOVAs. All significant main effects and interactions were broken down using the Scheffé test. Alpha was set at .05 for all analyses.

\section{Results}

\section{MIQ-R scores}

The Kruskal-Wallis test on the visual imagery scores revealed no significant difference between groups $[\mathrm{H}(3,179)=.32, p=.95]$. The Kruskal-Wallis test on the kinesthetic imagery scores revealed no significant difference between groups $[\mathrm{H}(3,179)=$ $.73, p=.87]$, (see Table 1 for details). These results did not show any differences in imagery abilities for all groups of participants (i.e., with and without the load in the TC or the AC condition).

\section{Movement time}


$2=3733.1, p<.01]$ and the action $[F(1,175)=74.66, p<.01]$. The ANOVA also revealed

The ANOVA revealed significant main effects of the distance of the paths $[F(2,350)$ significant interactions between the load and the action $[F(1,175)=6.14, p=.01]$; the distance of the paths and the action $[F(2,350)=31.24, p<.01]$; and the load, the distance of the paths, and the action $[F(2,350)=6.91, p<.01]$. The post hoc Newman-Keuls test revealed that irrespective of load or action conditions, the movement time increased with the distance of the paths $(p s=.0001)$. However, while the movement times were similar whatever the action (actual vs. imagined) and the load at the 5-m path distance, significant differences appeared at the 10-m and 15-m path distances, with shorter movement times for the imagined walking $(p s<.0001)$ and higher movement times in the loaded than in the unloaded condition for the actual actions only $(p s<.05)$ (Figure 1). No effect of the climatic conditions appeared in the present experiment ( $p s>.156)$.

\section{Isochrony index}

Please insert Figure 1 about here

The ANOVA revealed significant main effects of the load $[F(1,175)=17.64, p<.01$, $\left.\eta_{\mathrm{p}}^{2}=0.10\right]$, the climate $\left[F(1,175)=22.01, p<.01, \eta_{\mathrm{p}}^{2}=0.16\right]$ and the distance of the path $\left[F(2,350)=86.93, p<.01, \eta_{\mathrm{p}}{ }^{2}=0.20\right]$ as well as significant interactions between the load and the distance $\left[F(2,350)=12.88, p<.01, \eta_{\mathrm{p}}{ }^{2}=0.03\right]$ and between the climate and the distance $\left[F(2,350)=5.98, p<.01, \eta_{\mathrm{p}}{ }^{2}=0.02\right]$. The breakdown of the load and the distance interaction revealed that the isochrony index increased with the distance of the paths in both the loaded $(p s<.001)$ and the unloaded condition $(p s<.001$ except between $10-\mathrm{m}$ and $15-\mathrm{m}$, $p=.079)$. The isochrony index tended to be higher in the loaded than in the unloaded 
1 condition at 5 meters $(p=.06)$ and was significantly higher at the distances of $10(p=.01)$

2 and $15(p<.0001)$ meters.

3

4

5

Please insert Figure 2 about here

The breakdown of the climate and distance interaction revealed that the isochrony index increased with the distance of the paths in both the AC and the TC condition (ps < .0001). Moreover, although the isochrony index was higher in the TC than in the AC condition, the difference between these two conditions further increased for the distances of 10 and 15 meters $(p s<.001)$ (see Figure 3).

Please insert Figure 3 about here

\section{Discussion}

The aim of the present study was to evaluate the influence of climatic conditions on the mental chronometry and more precisely on the temporal characteristics of action simulation. Analyses were performed first on the duration of actual and imagined walking and second on the isochrony index, which reflects the temporal characteristics of motor imagery accuracy.

In accordance with the literature, our results confirmed that the actual and the imagined movement times increased with the distances of the paths (Guillot \& Collet, 2005; Guillot et al., 2012, for reviews) and conformed to Fitts' law (Cerritelli et al., 2000; Decety et al., 1989). However, our results partially disagreed with the temporal equivalence hypothesis between actual and imagined performance (Decety et al., 1989; Decety \& Michel, 1989; Maruff \& Velakoulis, 2000). Whereas temporal equivalence appeared for the 5-meter 
1 distance, confirming the results obtained in Papaxanthis et al. (2002) study using a 6-meter

2 distance walking task, significant differences appeared between the actual and the imagined

3 walking task for the distances of 10 and 15 meters. Indeed, for these longer distances, the imagined walking times were shorter than the actual walking times. The disparity between the results of the current experiment and those of Decety et al. (1989) study could be attributable to a distinct methodological procedure. Indeed, participants, in the latter study, were blindfolded. Elliot and Maraj (1994) proposed that the subjects' performance, in Decety et al. (1989) study, could reflect changes in memory function or in their space representation rather than any internal representation of the walking task. In addition, we can note that the underestimation of walking times during MI has been previously reported for long task durations (Grealy \& Sherer, 2008). The latter authors observed that motor imagery times were significantly shorter than actual walks times (at 15-meter, 33-meter and 65-meter) and suggested that the distance to be covered appeared to be an important factor in the equivalence of motor imagery timing. This result was also confirmed in Hanyu and Itsukushima (1995) study.

Moreover, this non-equivalence between the actual and the imagined performance for the longer distances was accentuated by the impact of the load, which did not slow down the duration of the imagined walking task, in contrast to the actual walking task. This difference between real and imagined movements durations, with the addition of a load, was largely reported in the literature (Cerritelli et al., 2000; Decety et al., 1989; Grealy \& Sherer, 2008; Guillot \& Collet, 2005). These findings suggested that temporal equivalence may be easier to observe for short distance, but when the distance and the difficulty of the task increases with the load, participants can have difficulty integrating some parameters of actions such as duration and force into movement simulation as previously observed with complex movements (Calmels \& Fournier, 2001). The previous explanatory hypothesis that states the 
1 impact of task difficulty on the temporal characteristics of the imagined walking task was

2 reinforced by the analysis performed on the isochrony index. Our findings revealed that the

3 value of the isochrony index increased with distance and that this increase was more

4 important for the loaded than for the unloaded condition. Consequently, these results

5 confirmed the results obtained in Cerritelli et al. (2000) study and the suggestion made in the

6 previous paragraph that the participants' ability to form accurate motor imagery decreases

7 when the constraints of the tasks increase, for example, by increasing the walking distance

8 (from 5 to 15 meters) and adding a load in a rucksack placed on their shoulders. Finally, as revealed in a subjective report, in Decety et al. (1989) study, we may also envisage that participants felt a marked rise of perceived effort when performing MI with a load and this feeling increased with the distance.

What about the impact of the climatic conditions on the mental chronometry? In the present experiment, no difference appeared between the actual and the imagined walking time as a function of climate (Tropical Climate vs. Air Conditioning). However, the TC had a negative impact on the imagery quality estimated with the mental chronometry, while no difference appeared with regard to the MIQ-R scores. We effectively observed a better isochrony index in the $\mathrm{AC}$ than in the $\mathrm{TC}$ condition. This result agrees with the literature, in which it is generally observed that a hot and wet environment leads to the decrement of cognitive performances such as vigilance, tracking, dual tasks or mental rotation (Berg et al., 2015; Gaoua, 2010; Qian et al., 2015; Robin et al., in press; Wetsel, 2011). Our results confirmed that motor imagery, conceived as the covert cognitive process of action production can also be negatively influenced by a TC. Importantly, the present experiment showed that the climate-induced effects were accentuated by the increase of the distance. Indeed, we observed that increasing the walking distances had deleterious effects on the capacity to form 
1 2 of action.

3

mental images that accurately integrate the temporal parameters of the mental representation

The results of the present study agree with the Maximal Adaptability Model (Hancock \& Warm, 1989), which assumes that heat exerts detrimental effects on performance by competing for and eventually draining attentional resources. Indeed, in a dual task realized at $30^{\circ} \mathrm{C}$, Chase, Karwowski, Benedict, Quesade, and Irwin-Chase (2001) observed that participants were unable to successfully allocate their attention to both tasks. It may be that when different sources of stress add up, for example, the temperature on the one hand and the difficulty of the task (i.e., combination of load and increase in walking distance) on the other hand, this consumption of resources leads to a decrease in motor imagery performance. In a tropical climate, which induces heat stress, motor imagery quality could then deteriorate because the total resource is insufficient, and the increase in distance represents a supplementary constraint.

Although we did not measure cerebral activity in the current experiment, neuroimagery studies revealed that similar neural structures (i.e., parietal cortex, supplementary motor area, premotor cortex, prefrontal cortex, cerebellum, primary motor cortex and spinal cord) are involved in MI and actual movement execution (Batula et al., 2017; Decety, 1996; Gerardin et al., 2000; Jeannerod, 2001). Recently, Cebolla et al. (2015) characterized the neural signature of MI (participants were asked to imagine throwing a tennis ball toward a target) using both event related potentials (ERP) and time frequency domain measurements (event related spectral perturbation and intertribal coherence). According to the authors, the representation of movement formed during MI might underlie a top-down process from the fronto-central areas reflected by the amplitude changes observed in fronto-central ERPs notably. In accordance with the latter study, Holmes and Calmels (2008) defined imagery as a top-down, knowledge driven process that involve the generation or re-generation 
1 of parts of neural network or brain representation (Cumming \& Williams, 2013). During MI

2 the imagers re-experience movements in their mind, on the basis of retrieved information

3 from long-term memory, the image being generated in working memory. Thus, given heat

4 alter mental process (e.g. working memory) and induces disturbances in frontal lobe function

5 (Gaoua, 2010; Gaoua et al. 2011) we could consider that TC would negatively influence

6 imagery quality. Further research measuring cerebral activity during MI, in TC, is needed.

Given the above explanation, one might wonder why all the factors that increased the level of difficulty of the task are not affected by the tropical climate. According to Papaxanthis et al. (2002) the activation of the same cerebral structures during MI and actual movement execution does not guarantee that all aspects of actual and imagined movement are similar. For example, in the current experiment, adding a load on the participants' shoulders decreased the isochrony index, but the decrease was similar under both the TC and AC. On the contrary, the detrimental influence of the TC effects were accentuated by the increase of the distance. These findings might indicate that the integration of force parameters and distance parameters into the duration of a movement simulation depends on different processes (Cerritelli et al., 2000; Decety et al., 1989; Grealy \& Sherer, 2008). Moreover it seems that heat stress more specifically influences the cognitive processes regulating the distance parameters in motor imagery. It may also be that simulated force parameters are cognitively less expensive than simulated distance parameters and that a longer heat exposure time is needed for the detrimental effect of the tropical climate (Ramsey \& Kwon, 1992) to affect the quality of motor imagery. Finally, it could be that the load facilitated the participants' body perception as it provided a kinaesthetic input. Further investigations are needed to clarify the varied effects of a tropical climate on motor imagery quality as a function of task constraints. 
This study is not without limitations. The evaluation of the influence of TC on MI should have been accompanied by the measures of the fatigue of the participants. Indeed, Qian et al. (2015) proposed that heat stress has a potential fatigue-enhancing effect when individual is performing highly cognition demanding attention task. In addition according to Lan and collaborators (2011), high level of relative humidity is known to increase feeling of fatigue. Further research using fatigue subjective estimation and an evaluation of the influence of relative humidity on MI are needed.

(1)

(1)
imagined walking may varied as a function of the difficulty of the task. Temporal congruence, revealed by the isochrony index, appeared for the simplest action, whereas it disappeared when the constraints of the task increased. Importantly, the difficulty of the task had deleterious effects on the participants' ability to build up the temporal characteristics of the imagined walking performance, and this effect was amplified when the performance was realized under tropical climate conditions.

\section{Conclusion}

The present study confirmed that the temporal congruence between actual walking and 
1 Calmels, C., Holmes, P., Lopez, E., Naman, V. (2006). Chronometric comparison of actual and imagined complex movement patterns. Journal of Motor Behavior, 38, 339-348.

Cebolla, A. M., Petieau, M., Cevallos, C., Leroy, A., Dan, B., \& Cheron, G. (2015). Longlasting cortical reorganization as the result of motor imagery of throwing a ball in a virtual tennis court. Frontiers in Psychology, 6, 1869. doi: 10.3389/fpsyg.2015. 01869

Cerritelli, B., Maruff, P., Wilson, P., \& Currie, J. (2000). The effect of external load on the force and timing components of mentally represented actions. Behavioral Brain Research, 108, 91-96.

Chase, B., Karwowski, W., Benedict, M., Quesade, P., \& Irwin-Chase, H. (2001). Effects of thermal stress on dual task performance and attention allocation. Ergonomics, under review. doi:10.1080/10803548.2003.11076550

Corcos, D. M. (1994). Temporal representation in the control of movement. Behavioural Brain Research, 17, 206.

Cumming, J., \& Williams, S. E. (2013). Introducing the revised applied model of deliberate imagery use for sport, dance, exercise and rehabilitation. Movement Sport Science, 82, 69-81. doi: $10.1051 / \mathrm{sm} / 2013098$

Debarnot, U., Sahraoui, D., Champely, S., Collet, C., \& Guillot, A. (2012). Selective influence of circadian modulation and task characteristics on motor imagery time. Research Quarterly for Exercise and Sport, 83, 442-50.

Decety, J. (1996). Do executed and imagined movements share the same central structures? Cognitive Brain Research, 3, 87-93.

Decety, J., Jeannerod, M., \& Prablanc, C. (1989). The timing of mentally represented actions. Behavioral Brain Research, 34, 35-42.

Decety, J., \& Michel, F. (1989). Comparative analysis of actual and mental movement times in two graphic tasks. Brain and Cognition, 11, 87-97. 
1 Elliott, D., \& Maraj, B. K. V. (1994). Visual-spatial movement goals. Behavioral and Brain Sciences, 17, 207.

Gaoua, N. (2010). Cognitive function in hot environments: A question of methodology. Scandinavian Journal of Medicine \& Science in Sports, 20, 60-70. doi:10.1111/j.1600-0838.2010.01210.x

Gaoua, N., Racinais, S., Grantham, J., \& El Massioui, F. (2011). Alterations in cognitive performance during passive hyperthermia are task dependent. International Journal of Hyperthermia, 27(1), 1-9. doi: 10.3109/02656736.2010.516305.

Gerardin E, Sirigu A, Lehericy S, Poline JB, Gaymard B, Marsault C, Agid Y, \& Le Bihan D. (2000). Partially overlapping neural networks for real and imagined hand movements. Cerebral Cortex, 10, 1093-1104.

Guillot, A., \& Collet, C. (2005). Duration of mentally simulated movement: a review. Journal of Motor Behavior, 37, 10-20.

Guillot, A., \& Collet, C. (2008). Construction of the motor imagery integrative model in sport: A review and theoretical investigation of motor imagery use. International Review of Sport and Exercise Psychology, 1, 31-44.

Guillot, A., Hoyek, N., Louis, M., \& Collet, C. (2012). Understanding the timing of motor imagery: Recent findings and future directions. International Review of Sport and Exercise Psychology, 5, 3-22. doi:10.1080/1750984X.2011.623787

Goss, S., Hall, C. R., Buckolz, E., \& Fishburne, G. J. (1986). Imagery ability and the acquisition and retention of movements. Memory and Cognition, 14, 469-477. doi:10.3758/BF03202518

Grealy, M.A., \& Shearer, G. F. (2008). Timing processes in motor imagery. European Journal of Cognitive Psychology, 20, 867-892. 
Hall, C., \& Martin, K. (1997). Measuring movement imagery abilities: A revision of the Movement Imagery Questionnaire. Journal of Mental Imagery, 21, 143-154.

Hancock, P., \& Vasmatzidis, I. (2003). Effects of heat stress on cognitive performance: The current state of knowledge. International Journal of Hyperthermia, 19, 355-372. doi:10.1080/0265673021000054630

Hancock, P. A., \& Warm, J. S. (1989). A dynamic model of stress and sustained attention. Human Factors, 31, 519-537. doi:10.7771/2327-2937.1024

Hanyu, K., \& Itsukushima, Y. (1995). Cognitive distance of stairways: Distance, traversal time and mental walking estimations. Environment and Behaviour, 27(4), 579-591.

Hanyu, K., \& Itsukushima, Y. (2000). Cognitive distance of stairways: A multi-stairway investigation. Scandinavian Journal of Psychology, 41, 63-69.

Holmes, P., \& Calmels, C. (2008). A neuroscientific review of imagery and observation use in sport. Journal of Motor Behavior, 40, 433-445.

Hue, O. (2011). The challenge of performing aerobic exercise in tropical environments: Applied knowledge and perspectives. International Journal of Sports Physiology and Performance, 6, 443-454.

Jeannerod, M. (2001). Neural simulation of action: a unifying mechanism for motor cognition. Neuroimage, 14, 103-109.

Jeannerod, M., \& Frak, V. (1999). Mental imaging of motor activity in humans. Current Opinion Neurobiology, 9, 735-739.

Lebon, F., Collet, C., \& Guillot, A. (2010). Benefits of motor imagery training on muscle strength. Journal of Strength and Conditioning, 24, 1680-1687. doi:10.1519/JSC.0b013e3181d8e936

Malouin, F., Richards, C. L., Jackson, P. L., \& Doyon, J. (2010). Motor imagery for optimizing the reacquisition of locomotor skills after cerebral damage. In A. Guillot \& 
C. Collet (Eds.), The neurophysiological foundations of mental and motor imagery (pp. 161-176). Oxford, UK: Oxford University Press.

Maruff, P., \& Velakoulis, D. (2000). The voluntary control of motor imagery. Imagined movements in individuals with feigned impairment and conversion disorder. Neuropsychologia, 38, 1251-1260.

Papaxanthis, C., Schieppati, M., Gentili, R., \& Pozzo, T. (2002). Imagined and actual arm movements have similar durations when performed under different conditions of directions and mass. Experimental Brain Research, 143, 447-452.

Pearson, J., Naselaris, T., Holmes, E. A., \& Kosslyn, S. M. (2015). Mental Imagery: Functional Mechanisms and Clinical Applications. Trends Cogition in Sciences, 19(10), 590-602. doi: 10.1016/j.tics.2015.08.003

Orliaguet, J. P., \& Coello, Y. (1998). Differences between actual and imagined putting movements in golf: A chronometric analysis. International Journal of Sport Psychology, 29, 157-169.

Qian, S., Li, M., Li, G., Liu, K., Li, B., Jiang, Q., Li, L., Yang, Z., \& Sun, G. (2015). Environmental heat stress enhances mental fatigue during sustained attention task performing: Evidence from an ASL perfusion study. Behavioral Brain Research, 280, 6-15. doi:10.1016/j.bbr.2014.11.036a

Ramsey, J., \& Kwon, Y. (1992). Recommended alert limits for perceptual motor loss in hot environments. International Journal of Industrial Ergonomics, 9, 245-257. doi:10.1016/0169-8141(92)90018-U

Robin, N., Coudevylle, R. G., Sinnapah, S., \& Hue, O. (in press). Effects of tropical climate on mental rotation: The role of imagery ability. American Journal of Psychology.

Robin, N., Dominique, L., Toussaint, L., Blandin, Y., Guillot, A., \& Le Her, M. (2007). Effects of motor imagery training on returning serve accuracy in tennis: The role of 
1

2

3

4

5

6 7

15

imagery ability. International Journal of Sport \& Exercise Psychology, 2, 177-188. doi:10.1080/1612197X.2007.9671818

Rulleau, T., Mauvieux, B., \& Toussaint, L. (2015). Influence of circadian rhythms on the temporal features of motor imagery for older adult inpatients. Archives of Physical Medicine and Rehabilitation, 96, 1229-1234. doi:10.1016/j.apmr.2015.02.015

Short, S. E., Tenute, A., \& Feltz, D. L. (2005). Imagery use in sport: Mediational effects for efficacy. Journal of Sports Sciences, 23, 951-960.

Wetsel, W. (2011). Hyperthermic effects on behavior. International Journal of Hyperthermia, 27, 353-373. doi:10.3109/02656736.2010.550905

Williams, E., Guillot, A., Di Rienzo, F., \& Cumming, J. (2015). Comparing self-report and mental chronometry measures of motor imagery ability. European Journal of Sport Science, 1-9. doi:10.1080/17461391.2015.1051133 
1 Table and Figures captions

2

3 Table 1

4 Medians (range) for the visual, and kinesthetic imagery scores as a function of the 5 experimental conditions used in the present experiment.

6

7 
1 Figure 1. Movement time (in seconds) as a function of distance (5,10 and 15 meters), load

2

3

4

5

6

7

8

9

10

11

12

13 condition (loaded vs. unloaded) and action (actual vs. imagined). I-beams indicates the $95 \%$ confidence intervals for the mean values.

Figure 2. Isochrony index (in seconds) as a function of distance (5, 10 and 15 meters) and load condition (loaded vs. unloaded). I-beams indicates the $95 \%$ confidence intervals for the mean values.

Figure 3. Isochrony index (in seconds) as a function of distance (5, 10 and 15 meters) and climatic condition (Tropical Climate vs. Air Conditioning). I-beams indicates the $95 \%$ confidence intervals for the mean values. 